CAOS 2018. The 18th Annual Meeting of the International Society for Computer Assisted Orthopaedic Surgery

\title{
Internal fixation in upper cervical spinal surgery: a randomized controlled study
}

\author{
Mingxing Fan ${ }^{1}$, Yajun Liu ${ }^{1}$ and Wei Tian ${ }^{1 *}$ \\ ${ }^{1}$ Department of Spine surgery, Beijing Jishuitan Hospital, Beijing, 100035, China \\ van0208@163.com, drtianweia@163.com
}

\begin{abstract}
Internal fixation in upper cervical spine $(\mathrm{C} 1$ and $\mathrm{C} 2)$ is a big challenge. There is a large anatomical variation, and the upper cervical region is adjacent to important organs such as spinal cords, oblongata, vertebral artery, nerve roots and other important structures, misplacement of internal fixation might lead not only to an instability of the screws, but also to neurological, vascular, and visceral injuries.

TianJi Robot was used in this study to compare the internal fixation safety and accuracy between using the navigation based robotic system and free-hand. In this randomized controlled study 51 patients were involved.

129 screws were smoothly implanted without intraoperative complications. In the robot-assisted surgery group, the analysis of the screw showed that 58 of 59 screws (98.3\%) were safely placed $(<2 \mathrm{~mm})$, and the mean deviation between the planned trajectory and the actual path is $1.14+/-0.09 \mathrm{~mm}$. In the free-hand fluoroscopy-guided surgery and navigation assisted surgery group, assessment of screw accuracy showed that 66 of 70 screws $(94.3 \%)$ were safely placed $(<2 \mathrm{~mm})$, and the mean deviation between the planned trajectory and the actual path is $1.48+/-0.14 \mathrm{~mm}$. There was no statistical difference in Gertzbein-Robbins classification distribution between these two groups ( $\mathrm{P}$ value $=0.551)$, however, the discrepancies between the actual path and planned trajectory in this two groups have statistical difference ( $\mathrm{P}$ value $=0.047$ ).

The TianJi Robot has demonstrated its safety and accuracy in upper cervical spinal
\end{abstract}


surgery upon free-hand technic and the navigation technic, which will have expanded applications in spinal surgery.

\section{Introduction}

Internal fixation in upper cervical spine $(\mathrm{C} 1$ and $\mathrm{C} 2)$ is a big challenge. There is a large anatomical variation, the collection of different studies shows that the same upper cervical vertebral segments in different individuals, the height and width of the cervical pedicle, the axial projection point and the axis angle of the cervical pedicle have great difference. The height of cervical pedicle ranges from 5.1 to 9.5 $\mathrm{mm}$, and the width ranges from 3 to $7.5 \mathrm{~mm}[1,2]$. And the upper cervical region is adjacent to important organs such as spinal cords, oblongata, vertebral artery, nerve roots and other important structures, misplacement of internal fixation might lead not only to a instability of the screws, but also to neurological, vascular, and visceral injuries.

The image based computer assisted navigation system has been applied into upper cervical spinal surgery to improve the screw insertion accuracy, by register the patient's fluoroscopy images into the navigation system, surgeons could actually "see" the upper cervical and the neighboring important hidden structures during the surgery. However, there are still questions about the benefits of the navigation system have[3]. On the other hand, the surgeons' force control and steadiness still insufficient to face the challenge of complicated upper cervical spinal surgery.

One way to overcome the above drawbacks and facing the challenge of the upper cervical spinal surgeries is to use a navigation based robotic system. In this study, we introduced a navigation based robotic system which could do upper cervical surgery and compared the internal fixation safety and accuracy between using the navigation based robotic system and using only free-hand or navigation.

\section{Materials and methods}

TianJi Robot ${ }^{\circledR}$ was used in this study, which was co-designed and modified by Beijing Jishuitan Hospital and Beijing Tinavi company. It was a robot-assisted surgical navigation device based on 3D fluoroscopy, and it is the only orthopaedics robot that can do upper cervical spinal surgery $[4,5]$.

This randomized controlled study which was approved by the Ethics Committee, 51 patients were involved and all has been fully informed and sign the informed consent from Aug. 2015 to Dec. 2017. 27 patients were treated by robot-assisted upper cervical spinal surgery, and 24 patients were treated by free-hand fluoroscopy-guided surgery or navigation assisted surgery. After surgery, a postoperative CT 
scan was performed and analyzed. The CT image data were reconstructed in sagittal, coronal and axial views by the mimics 17.0 software, and a blind evaluation of the position of the screws was performed by two spine surgeons who were not involved in this study. Any penetration of the cortex in the lateral, medial, cranial or caudal directions was measured according to the Gertzbein-Robbins classification[6] (A: no cortical violation; B: cortical breach $<2 \mathrm{~mm}$; $\mathrm{C}: \geq 2 \mathrm{~mm}$ to $<4 \mathrm{~mm}$; D: $\geq 4 \mathrm{~mm}$ to $<6 \mathrm{~mm}$; E: $\geq 6$ $\mathrm{mm})$. Also, we measured the discrepancies between the actual path and planned trajectory[7].

\section{Results}

In all 51 surgeries, 129 screws were smoothly implanted without intraoperative complications and all patients were discharged for rehabilitation after surgery. There were no differences between these two groups in the baseline characteristics. In the robot-assisted surgery group, the analysis of the screw showed that 58 of 59 screws $(98.3 \%$ ) were safely placed ( $<2 \mathrm{~mm}$, Gertzbein-Robbins category A+B, i.e., good screw positions in Fig. 1), 1 screw fall in category $\mathrm{C}$, and the mean deviation between the planned trajectory and the actual path is $1.14+/-0.09 \mathrm{~mm}$. In the free-hand fluoroscopy-guided surgery and navigation assisted surgery group, assessment of screw accuracy showed that 66 of 70 screws (94.3\%) were safely placed $(<2 \mathrm{~mm}$, Gertzbein-Robbins category A+B), 4 screws fall in category $\mathrm{C}$, and the mean deviation between the planned trajectory and the actual path is $1.48+/-0.14 \mathrm{~mm}$.

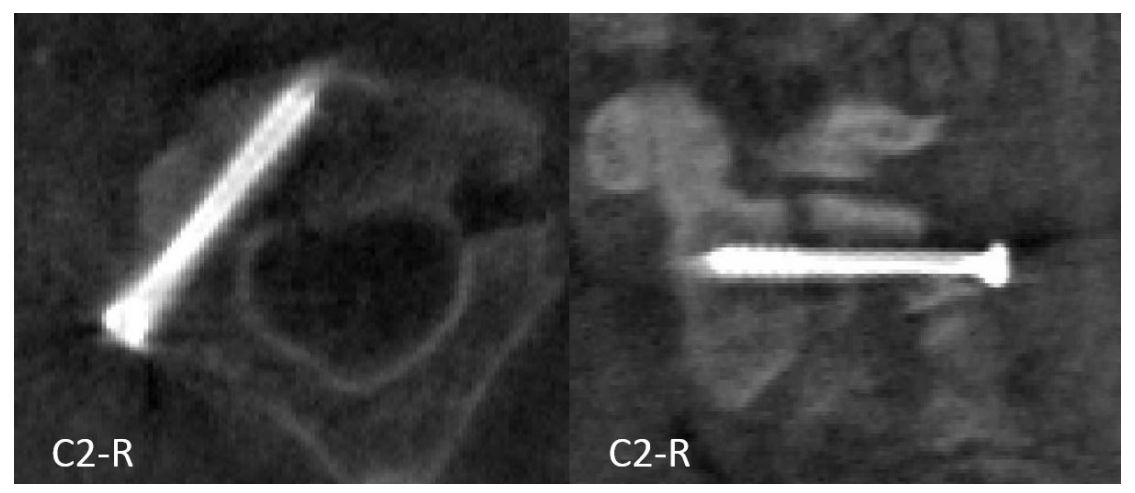

Figure 1: Postoperative computer tomography scans indicating the results of the $\mathrm{C} 2$ screw for safety analysis.

There was no statistical difference in Gertzbein-Robbins classification distribution between these two groups $(\mathrm{P}$ value $=0.551)$, however, the discrepancies between the actual path and planned trajectory in this two groups have statistical difference (P value $=0.047$, Fig. 2 ). 


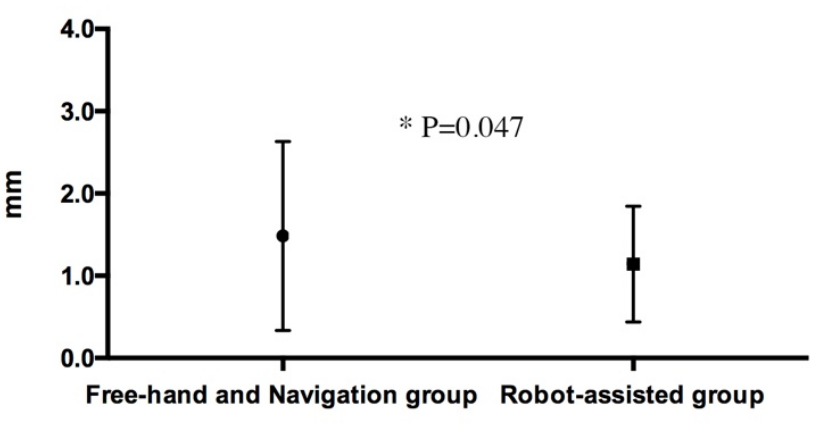

Figure 2: The discrepancies between the actual path and planned trajectory in free-hand and navigation group and robot-assisted group

\section{Discussion}

Internal fixation in upper cervical spine can be technically challenging because of the difficult approach and complex morphology of the vertebrae and surrounding vital structures. Although the navigation system was used, still failed to achieve satisfactory safety and accuracy. As the navigation and robot techniques are becoming more and more popular, one way to improve the safety and accuracy is to use a robot. The Renaissance and the ROSA Spine both shows the remarkable results in clinical applications $[8,9]$, but the limitation is that they both cannot do the cervical spinal surgery. The atlantoaxial fixation robot brought by Bertelsen was not sufficient for clinical use[10]. The TianJi Robot ${ }^{\circledR}$ that we co-designed was demonstrated its safety and accuracy in upper cervical spinal surgery upon the free-hand technic and the navigation technic, which will have expanded applications in spinal surgery.

\section{References}

[1] H.S. An, J.J. Wise, R. Xu, Anatomy of the cervicothoracic junction: a study of cadaveric dissection, cryomicrotomy, and magnetic resonance imaging, Journal of spinal disorders 12(6) (1999) $519-25$.

[2] N.A. Ebraheim, R. Xu, T. Knight, R.A. Yeasting, Morphometric evaluation of lower cervical pedicle and its projection, Spine 22(1) (1997) 1-6.

[3] R. Verma, S. Krishan, K. Haendlmayer, A. Mohsen, Functional outcome of computer-assisted 
spinal pedicle screw placement: a systematic review and meta-analysis of 23 studies including 5,992 pedicle screws, European spine journal : official publication of the European Spine Society, the European Spinal Deformity Society, and the European Section of the Cervical Spine Research Society 19(3) (2010) 370-5.

[4] W. Tian, H. Wang, Y.J. Liu, Robot-assisted Anterior Odontoid Screw Fixation: A Case Report, Orthopaedic surgery 8(3) (2016) 400-4.

[5] W. Tian, Robot-Assisted Posterior C1-2 Transarticular Screw Fixation for Atlantoaxial Instability: A Case Report, Spine 41 Suppl 19 (2016) B2-B5.

[6] I. Pechlivanis, G. Kiriyanthan, M. Engelhardt, M. Scholz, S. Lucke, A. Harders, K. Schmieder, Percutaneous placement of pedicle screws in the lumbar spine using a bone mounted miniature robotic system: first experiences and accuracy of screw placement, Spine 34(4) (2009) 392-8.

[7] S. Kim, J. Chung, B.J. Yi, Y.S. Kim, An assistive image-guided surgical robot system using Oarm fluoroscopy for pedicle screw insertion: preliminary and cadaveric study, Neurosurgery 67(6) (2010) 1757-67; discussion 1767.

[8] L. Chenin, J. Peltier, M. Lefranc, Minimally invasive transforaminal lumbar interbody fusion with the ROSA(TM) Spine robot and intraoperative flat-panel CT guidance, Acta neurochirurgica 158(6) (2016) 1125-8.

[9] D.P. Devito, L. Kaplan, R. Dietl, M. Pfeiffer, D. Horne, B. Silberstein, M. Hardenbrook, G. Kiriyanthan, Y. Barzilay, A. Bruskin, D. Sackerer, V. Alexandrovsky, C. Stuer, R. Burger, J. Maeurer, G.D. Donald, R. Schoenmayr, A. Friedlander, N. Knoller, K. Schmieder, I. Pechlivanis, I.S. Kim, B. Meyer, M. Shoham, Clinical acceptance and accuracy assessment of spinal implants guided with SpineAssist surgical robot: retrospective study, Spine 35(24) (2010) 2109-15.

[10] A. Bertelsen, J. Melo, E. Sanchez, D. Borro, A review of surgical robots for spinal interventions, The international journal of medical robotics + computer assisted surgery : MRCAS 9(4) (2013) 40722. 